\title{
An Interpretation of SDF Based Performance Measures
}

\author{
Paul Söderlind*
}

December 3, 1999

\begin{abstract}
This note discusses stochastic discount factor (SDF) measures of mutual fund performance. It shows that the most common SDF performance measures can be interpreted as Jensen's "alphas."

Keywords: mean-variance intersection, managed portfolios, conditional alphas and betas

JEL Classification Numbers: G11, G12, G23
\end{abstract}

View $\log$ file

View source file

\section{Introduction}

Mutual fund performance evaluation is often done in terms of the intercept ("alpha") in a linear regression of (a vector of) fund excess returns, $r_{2 t}$, on a vector of benchmark excess returns, $r_{1 t}$. In Huberman and Kandel's (1987) model

$$
r_{2 t}=\alpha+\beta r_{1 t}+\varepsilon_{t}, \mathrm{E} \varepsilon_{t}=\mathbf{0} \text { and } \operatorname{Cov}\left(r_{1 t}, \varepsilon_{t}\right)=\mathbf{0},
$$

neutral performance (mean variance intersection) requires $\alpha=\mathbf{0}$.

* Stockholm School of Economics and CEPR. Address: Stockholm School of Economics, PO Box 6501, SE-113 83 Stockholm, Sweden. E-mail: Paul.Soderlind@hhs.se. 
Recently, performance evaluations have often been cast in terms of an $\mathrm{SDF}, m_{t}$. The canonical asset pricing model $\mathrm{E}_{t-1} r_{1 t} m_{t}=\mathbf{0}$ implies

$$
\mathrm{E} z_{1 t-1} \otimes r_{1 t} m_{t}=\mathbf{0}
$$

where $z_{1 t-1}$ is a vector of instruments known in $t-1$, and $\otimes$ denotes a Kronecker product. Method 1 in (Chen and Knez 1996) (henceforth CK) is essentially the following when cast in excess returns: First, estimate a candidate SDF as in (Hansen and Jagannathan 1991): $\hat{m}_{t}=\bar{m}+\left(z_{1 t-1} \otimes\right.$ $\left.r_{1 t}-\mathrm{E} z_{1 t-1} \otimes r_{1 t}\right)^{\prime} \theta$, where $\bar{m}$ is a constant (the inverse of the expected gross return on a riskfree asset) and the vector $\theta$ is chosen to satisfy the sample analogue to (2). Second, test if this SDF "prices" the mutual funds returns, $r_{2 t}$, that is, if $\mathrm{E} \lambda_{2 t}=\mathbf{0}$ in

$$
\mathrm{E} \lambda_{2 t}=\mathrm{E} z_{2 t-1} \otimes r_{2 t} \hat{m}_{t}
$$

where $z_{2 t-1}$ also is a vector of instruments known in $t-1\left(z_{2 t}=z_{1 t}\right.$ in $\left.\mathrm{CK}\right)$. If not, this is interpreted as non-neutral performance, and the sample average of $\lambda_{2 t}$ (possibly multiplied with the gross riskfree return; see below) is taken to be the SDF performance measure.

(Ferson 1995) and (Bekaert and Urias 1996) show that the restrictions needed for neutral performance in (3) are the same as a generalized version of Huberman and Kandel's (1987) mean variance (MV) intersection and that the tests are identical. 1

\footnotetext{
${ }^{1}$ In practice, the SDF test is done within the GMM framework. The sample analogues of both (2) and (3) are used as moment conditions to estimate the $\theta$-coefficients in the SDF (this is Method 2 in CK). There are then more moment conditions than parameters (the difference equals the number of elements in $\lambda_{2 t}$ ), and the SDF test of neutral performance amounts to testing if all moments conditions are satisfied at the same vector $\theta$. The advantage of this method is that it accounts for the uncertainty about $\theta$; the disadvantage is that it does not generate a performance measure. See, among others, (Ferson 1995), (Chen and Knez 1996), (Bekaert and Urias 1996), and (Dahlquist and Söderlind 1999) for analysis of the properties of such tests.
} 


\section{Relation Between the SDF Performance Mea- sure and Jensen's "Alpha"}

We know that a neutral performance according to the SDF measure, $\mathrm{E} \lambda_{2 t}=$ 0 , is the same as a zero alpha. But what happens when the performance is non-neutral? Are the SDF measure and the alpha still they same, and does the sample average of $\lambda_{2 t}$ have a meaningful economic interpretation? Given duality between MV frontiers and the linear SDF (see (Hansen and Jagannathan 1991)), it should not come as big surprise that the two performance measures are very similar. This is demonstrated in the following proposition.

Proposition 1 Let $\mathfrak{R}_{1 t}$ be vector of payoffs of some benchmarks assets with prices $q_{1 t}$. Construct a stochastic discount factor as in (Hansen and Jagannathan 1991): $m_{t}=\bar{m}+\left(\Re_{1 t}-E \Re_{1 t}\right)^{\prime} \theta$, where $\bar{m}$ is a constant and $\theta$ is chosen to make $m_{t}$ "price" $\mathfrak{R}_{1 t}$, that is, so $E \mathfrak{R}_{1 t} m_{t}=E q_{1 t}$. Consider another set of assets with payoffs $\mathfrak{R}_{2 t}$, prices $q_{2 t}$, and SDF-performance

$$
E \lambda_{2 t}=E \Re_{2 t} m_{t}-E q_{2 t}
$$

Let the factor portfolio model be the linear regression

$$
\mathfrak{R}_{2 t}=\alpha+\beta \Re_{1 t}+\varepsilon_{t} \text {, where } E \varepsilon_{t}=\mathbf{0} \text { and } \operatorname{Cov}\left(\Re_{1 t}, \varepsilon_{t}\right)=\mathbf{0} \text {. }
$$

Then, the relation between $E \lambda_{2 t}$ and $\alpha$ is

$$
E \lambda_{2 t} / \bar{m}=\alpha-\left(E q_{2 t}-\beta E q_{1 t}\right) / \bar{m}
$$

Proof. It follows directly that $\theta=\operatorname{Var}\left(\mathfrak{R}_{1 t}\right)^{-1}\left(\mathrm{E} q_{1 t}-\mathrm{E} \mathfrak{R}_{1 t} \bar{m}\right)$. Using this and the expression for $m_{t}$ in (4) gives

$$
\mathrm{E} \lambda_{2 t}=\mathrm{E} \Re_{2 t} \bar{m}+\operatorname{Cov}\left(\Re_{2 t}, \Re_{1 t}\right) \operatorname{Var}\left(\Re_{1 t}\right)^{-1}\left(\mathrm{E} q_{1 t}-\mathrm{E} \Re_{1 t} \bar{m}\right)-\mathrm{E} q_{2 t} .
$$


We now rewrite this equation in terms of the parameters in the factor portfolio model (5). The latter implies $\mathrm{E} \mathfrak{R}_{2 t}=\alpha+\beta \mathrm{E} \mathfrak{R}_{1 t}$, and the least squares estimator of the slope coefficients are $\beta=\operatorname{Cov}\left(\mathfrak{R}_{2 t}, \mathfrak{R}_{1 t}\right) \operatorname{Var}\left(\mathfrak{R}_{1 t}\right)^{-1}$. Using these two facts in (7) gives (6).

Remark 2 The sample analogues to (4)-(6) are obtained by replacing the population moments with sample moments.2

\section{Two Examples}

To illustrate Proposition 11 we take a look at two examples where $\mathfrak{R}_{1 t}$ and $\mathfrak{R}_{2 t}$ are excess returns scaled by some instruments known in $t-1$, that is, when $\mathfrak{R}_{i t}=z_{i t-1} \otimes r_{i t}$ and $\mathrm{E} q_{i t}=\mathbf{0}$.

\subsection{Unconditional Performance}

Suppose $z_{2 t}$ is a constant. Equations (4)-(6) are then (with superscript ${ }^{u}$ added)

$$
\begin{aligned}
& \mathrm{E} \lambda_{2 t}^{u}=\mathrm{E} r_{2 t} m_{t}, \\
& r_{2 t}=\alpha^{u}+\beta^{u}\left(z_{1 t-1} \otimes r_{1 t}\right)+\varepsilon_{t}^{u}, \text { and } \\
& \mathrm{E} \lambda_{2 t}^{u} / \bar{m}=a^{u} .
\end{aligned}
$$

The first equation can be rearranged as

$$
\mathrm{E} r_{2 t}=-\operatorname{Cov}\left(r_{2 t}, m_{t}\right) / \bar{m}+\mathrm{E} \lambda_{2 t}^{u} / \bar{m} .
$$

\footnotetext{
${ }^{2}$ In a recent paper (He, $\mathrm{Ng}$, and Zhang 1999) argues that the benchmark returns, $\mathfrak{R}_{1 t}$, can only be a subset of the returns in the investment opportunity set, $\mathfrak{R}_{0 t}$. The SDF in Proposition 11 will typically not be able to price $\mathfrak{R}_{0 t}$ : $\mathrm{E} \lambda_{0 t}=\mathrm{E} \mathfrak{R}_{0 t} m_{t}-\mathrm{E} q_{0 t}$ may be non-zero. They therefore suggest using an SDF $m_{t}^{*}=\bar{m}+\left(\mathfrak{R}_{1 t}-\mathrm{E} \mathfrak{R}_{1 t}\right)^{\prime} \theta^{*}$, where $\theta^{*}$ is chosen to minimize the sum of squared pricing errors of $\mathfrak{R}_{0 t}$ (not of $\mathfrak{R}_{1 t}$ as in Proposition 11. It is straightforward to show that $\theta^{*}=\theta-\left(\Sigma_{01}^{\prime} \Sigma_{01}\right)^{-1} \Sigma_{01}^{\prime} \mathrm{E} \lambda_{0 t}$, where $\Sigma_{i j}$ is the covariance matrix of $\mathfrak{R}_{i t}$ and $\mathfrak{R}_{j t}$. Clearly, the two vectors of coefficients coincide if $\mathrm{E} \lambda_{0 t}=0$. It can also be shown that the performance of a fund using $m_{t}^{*}$ is $\mathrm{E} \lambda_{2 t}^{*}=\mathrm{E} \lambda_{2 t}-$ $\Sigma_{21}\left(\Sigma_{01}^{\prime} \Sigma_{01}\right)^{-1} \Sigma_{01}^{\prime} \mathrm{E} \lambda_{0 t}$. This shows, for instance, that $\mathrm{E} \lambda_{2 t}^{*}$ is likely to be less than $\mathrm{E} \lambda_{2 t}$ if the fund is positively correlated with a benchmark, which in turn is positively correlated with an asset which the SDF in Proposition 1 assigned too good performance.
} 
The expected excess return of a fund equals the risk premium implied by the SDF (the covariance term) plus the average (unconditional) performance, $\mathrm{E} \lambda_{2 t}^{u} / \bar{m}$. The multiplication of $\mathrm{E} \lambda_{2 t}^{u}$ by $1 / \bar{m}$ transforms the performance measure into a per period return (recall that $1 / \bar{m}$ is the average gross return on a riskfree asset).

The second and third equations in (8) show that the unconditional performance measure, $E \lambda_{2 t}^{u} / \bar{m}$, is the alpha in the factor portfolio model with time-varying betas. The reason is that $\beta\left(z_{1 t-1} \otimes r_{1 t}\right)$ can be written on the form $z_{1 t-1}^{\prime} \varphi r_{1 t}$, where $z_{1-1}^{\prime} \varphi$ can be thought of as time-varying "betas." Such models have been used in, for instance, (Ferson and Schadt 1996).

\subsection{Conditional Performance}

Suppose now that $z_{2 t}$ is vector of stochastic information variables. In this case, (4)-(6) are (with superscript ${ }^{c}$ added)

$$
\begin{aligned}
& \mathrm{E} \lambda_{2 t}^{c}=\mathrm{E} z_{2 t-1} \otimes r_{2 t} m_{t}, \\
& z_{2 t-1} \otimes r_{2 t}=\alpha^{c}+\beta^{c}\left(z_{1 t-1} \otimes r_{1 t}\right)+\varepsilon_{t}^{c}, \text { and } \\
& \mathrm{E} \lambda_{2 t}^{c} / \bar{m}=a^{c} .
\end{aligned}
$$

Let $\lambda_{2 t}^{u} / \bar{m}=r_{2 t} m_{t} / \bar{m}$, which can be thought of as the risk adjusted excess return, or performance, in time $t$ : recall from (8)-(9) that $\mathrm{E} \lambda_{2 t}^{u} / \bar{m}$ measures average performance. With this definition, we can rewrite the first equation in 10$)$ as

$$
\mathrm{E} \lambda_{2 t}^{c} / \bar{m}=\mathrm{E} z_{2 t-1} \otimes \lambda_{2 t}^{u} / \bar{m}
$$

The conditional performance measure, $\mathrm{E} \lambda_{2 t}^{c} / \bar{m}$, is a vector which shows to what extent the risk adjusted return in $t$ can be forecasted by each of the information variables in $z_{2 t-1}$ (if $z_{2 t-1}$ or $\lambda_{2 t}^{u}$ has zero mean then $\mathrm{E} \lambda_{2 t}^{c} / \bar{m}$ is a vector of covariances). Non-neutral conditional performance can be exploited by dynamically buying and selling shares in mutual funds. 
The second and third equations in 10 show that the vector of conditional performance measures, $E \lambda_{2 t}^{c} / \bar{m}$, is the vector the alphas in a factor portfolio model with time-varying betas for "managed portfolios" of funds. This follows from that the left hand side in the factor model can be thought of as portfolios consisting of different funds, where the portfolio weights change in response to movements in the information variables $z_{2 t-1} !^{3}$

\section{Conclusions}

This note shows that the SDF measures of fund performance in (Chen and Knez 1996) are the same as the performance measures from a factor portfolio model with time-varying betas: the unconditional SDF measure corresponds to a Jensen's alpha for a fund, and the conditional SDF measures to a vector of Jensen's alphas for managed portfolios of funds.

\section{References}

Bekaert, G., and M. S. Urias, 1996, "Diversification, integration and emerging market closed-end funds," Journal of Finance, 51, 835-869.

Chen, Z., and P. J. Knez, 1996, "Portfolio measurement: theory and applications," Review of Financial Studies, 9, 511-555.

Christopherson, J. A., W. E. Ferson, and D. A. Glassman, 1998, "Conditioning manager alphas on economic information: another look at the persistence of performance," Review of Financial Studies, 11, 111-142.

\footnotetext{
${ }^{3}$ This is similar, but not identical to a "conditional alpha" model, $r_{2 t}=\alpha+\eta \tilde{z}_{2 t-1}+$ $\beta\left(z_{1 t-1} \otimes r_{1 t}\right)+\varepsilon_{t}$, where $\eta \neq \mathbf{0}$ is interpreted as a time varying alpha (see, for instance, (Christopherson, Ferson, and Glassman 1998)). The conditional alpha model can be thought of as a linearized version of the conditional performance test.
} 
Dahlquist, M., and P. Söderlind, 1999, "Evaluating Portfolio Performance with Stochastic Discount Factors," Journal of Business, 72, 347-383.

Ferson, W. E., 1995, "Theory and empirical testing of asset pricing models," in Robert A. Jarrow, Vojislav Maksimovic, and William T. Ziemba (ed.), Handbooks in Operations Research and Management Science . pp. 145-200, North-Holland, Amsterdam.

Ferson, W. E., and R. Schadt, 1996, "Measuring fund strategy and performance in changing economic conditions," Journal of Finance, 51, 425-461.

Hansen, L. P., and R. Jagannathan, 1991, "Implications of security market data for models of dynamic economies," Journal of Political Economy, 99, $225-262$.

He, J., L. Ng, and C. Zhang, 1999, "Asset Pricing Specification Errors and Performance Evaluation," Mimeo, Chinese University of Hong Kong.

Huberman, G., and S. Kandel, 1987, "Mean-variance spanning," Journal of Finance, 42, 873-888. 